\title{
ADDITIVE AVERAGE SCHWARZ WITH ADAPTIVE COARSE SPACES: SCALABLE ALGORITHMS FOR MULTISCALE PROBLEMS*
}

\author{
LESZEK MARCINKOWSKI ${ }^{\dagger}$ AND TALAL RAHMAN ${ }^{\ddagger}$
}

\begin{abstract}
We present an analysis of the additive average Schwarz preconditioner with two newly proposed adaptively enriched coarse spaces, which were presented at the twenty-third international conference on domain decomposition methods in Korea, for solving second-order elliptic problems with highly varying and discontinuous coefficients. It is shown that the condition number of the preconditioned system is bounded independently of the variations and the jumps in the coefficient while depending only on a prescribed threshold for the eigenvalues of the coarse space, and it depends linearly on the mesh parameter ratio $\mathrm{H} / \mathrm{h}$ that is the ratio between the subdomain size and the mesh size thereby retaining the same optimality and scalability of the original additive average Schwarz preconditioner.
\end{abstract}

Key words. domain decomposition preconditioner, additive average Schwarz method, adaptive coarse space, multiscale finite element

AMS subject classifications. 65N55, 65N30, 65N22, 65F08

1. Introduction. Additive Schwarz methods are considered among the most effective preconditioners for solving algebraic systems arising from the discretization of elliptic partial differential equations. They generate algorithms that are easy to implement, inherently parallel, scalable, and fast. With proper enrichment of the coarse spaces, the methodology has recently been quite successfully applied to multiscale problems with highly heterogeneous and varying coefficients, the class of problems which most standard iterative solvers have difficulty to solve efficiently. Additive average Schwarz is one of the simplest of all additive Schwarz preconditioners because it is easy to construct and quite straightforward to analyze. Unlike most additive Schwarz preconditioners, its local subspaces are defined on non-overlapping subdomains, and it requires no explicit coarse grid as its coarse space is simply defined as the range of an averaging operator. By enriching its coarse space with functions corresponding to the bad eigenvalues of the local stiffness matrix, it has been shown numerically in a recent presentation, cf. [28], that the method can be made both scalable and robust with respect to any variation and jump in the coefficient when solving multiscale problems. The purpose of this paper is to give a complete analysis of the method presented in that paper.

The additive average Schwarz method in its original form was first introduced for secondorder elliptic problems in [3], where the method was applied to and analyzed for problems with constant coefficients in each subdomain and jumps only across subdomain boundaries. The method was further extended to non-matching grids using the mortar discretization in $[2,32,33]$ and to fourth-order problems in [15]. For multiscale problems where the coefficient may be highly varying and discontinuous also inside subdomains, the method has been analyzed in [12], where it has been shown that the condition number of the preconditioned system depends linearly on the jumps of the coefficient in the layers of the subdomains and quadratically on the ratio of the coarse to the fine mesh parameters. The method has very recently been extended to the Crouzeix-Raviart finite volume discretization, cf. [25, 26], showing similar results. All these results on the additive average Schwarz method, however, suggest that the method by itself cannot be robust for multiscale problems unless some form

\footnotetext{
* Received September 26, 2017. Accepted February 2, 2018. Published online on April 12, 2018. Recommended by Marcus Sarkis.

${ }^{\dagger}$ Faculty of Mathematics, University of Warsaw, Banacha 2, 02-097 Warszawa, Poland (Leszek. Marcinkowski@mimuw. edu.pl).

${ }^{\ddagger}$ Faculty of Engineering and Science, Western Norway University of Applied Sciences, Inndalsveien 28, 5063 Bergen, Norway (Talal. Rahman@hvl.no).
} 
of enrichment of the coarse space is made. This eventually led the research to the approach recently presented at the twenty-third international conference on domain decomposition, cf. [28], where adaptively chosen eigenfunctions of certain local eigenvalue problems extended by zero to the rest of the domain, are added to the standard average Schwarz coarse space. This idea of enriching the coarse space with eigenfunctions for improved convergence goes back several years; cf., e.g., the paper $[4,5]$ on a substructuring domain decomposition method and [9] on an algebraic multigrid method.

A systematic study of the performance of domain decomposition preconditioners for multiscale partial differential equations with respect to the contrast in the coefficients was started in $[1,19]$. There the use of some multiscale finite element method for the coarse problem was introduced, but the coarse space was not enriched. Using the idea of enrichment to solve multiscale problems started only very recently with the papers [16, 17, 29]. Since then, a number of other works have emerged proposing algorithms based on solving different eigenvalue problems; see, e.g., $[10,11,13,14,18,36]$ for those using the additive Schwarz framework for their algorithms and [8,20,21, 22, 23, 24, 27, 30, 31, 35] for those using the FETI-DP or the BDDC framework for their algorithms.

Throughout this paper, we use the following notations: $x \lesssim y$ and $w \gtrsim z$ denote that there exist positive constants $c$ and $C$ independent of the mesh parameters $h$ and $H$ and the jump of the coefficients such that $x \leq c y$ and $w \geq C z$, respectively.

The remainder of the paper is organized as follows: we state the discrete problem in Section 2, the additive Schwarz method in Section 3, and the coarse spaces in Section 4. In Section 5 a condition number bound is given and proved. Some numerical results are then presented in Section 6.

2. Discrete problem. Let $\Omega$ be a polygonal domain in the plane. We consider a model multiscale elliptic problem: Find $u^{*} \in H_{0}^{1}(\Omega)$ such that

$$
a\left(u^{*}, v\right)=\int_{\Omega} f v d x, \quad v \in H_{0}^{1}(\Omega)
$$

where

$$
a(u, v)=\int_{\Omega} \alpha(x) \nabla u \nabla v d x,
$$

$f \in L^{2}(\Omega)$, and $\alpha \in L^{\infty}(\Omega)$ is a positive coefficient function. We assume that there exists an $\alpha_{0}>0$ such that $\alpha(x) \geq \alpha_{0}$ in $\Omega$. Since we can scale the problem by $\alpha_{0}^{-1}$, we can further assume that $\alpha_{0}=1$.

We introduce the triangulation $T_{h}(\Omega)=T_{h}=\{\tau\}$ consisting of the triangles $\tau$ and assume that this triangulation is quasi-uniform in the sense of $[6,7]$. Let $V^{h}$ be the discrete finite element space consisting of continuous piecewise linear functions with zero on the boundary $\partial \Omega$, i.e.,

$$
V^{h}=\left\{v \in C(\bar{\Omega}): v_{\mid \tau} \in P_{1}(\tau), \quad \tau \in T_{h}, v=0 \text { on } \partial \Omega\right\},
$$

where $P_{1}(\tau)$ is the space of linear polynomials over the triangle $\tau \in T_{h}$. Each $v \in V^{h}$ can be represented in the standard nodal basis as $v=\sum_{x \in N_{h}} v(x) \phi_{x}$, where $N_{h}$ is the set of the nodal points, i.e., all vertices of triangles in $T_{h}$ which are not on $\partial \Omega$.

The corresponding discrete problem is then to find $u_{h}^{*} \in V^{h}$ such that

$$
a\left(u_{h}^{*}, v\right)=\int_{\Omega} f v d x, \quad v \in V^{h} .
$$


The Lax-Milgram theorem yields that this problem has a unique solution.

Note that for $u, v \in V^{h}$, their gradients are constant over each triangle $\tau \in T_{h}$. Thus we see that $\int_{\tau} \alpha(x) \nabla u \nabla v d x=\left(\nabla u_{\mid \tau}\right)\left(\nabla v_{\mid \tau}\right) \int_{\tau} \alpha(x) d x$. Hence without any loss of generality, we may assume that $\alpha$ is piecewise constant over the triangles of the triangulation $T_{h}$.

Further we assume that we have a coarse partition of $\Omega$ into open connected polygonal subdomains $\left\{\Omega_{k}\right\}_{k=1}^{N}$ such that $\bar{\Omega}=\bigcup_{k=1}^{N} \bar{\Omega}_{k}$, where each $\bar{\Omega}_{k}$ is a sum of some closed triangles of $T_{h}$. Let $H=\max _{k=1, \ldots, N} \operatorname{diam}\left(\Omega_{k}\right)$ be the coarse mesh parameter and $\Gamma$ the interface defined as $\Gamma=\bigcup_{k=1}^{N} \partial \Omega_{k} \backslash \partial \Omega$.

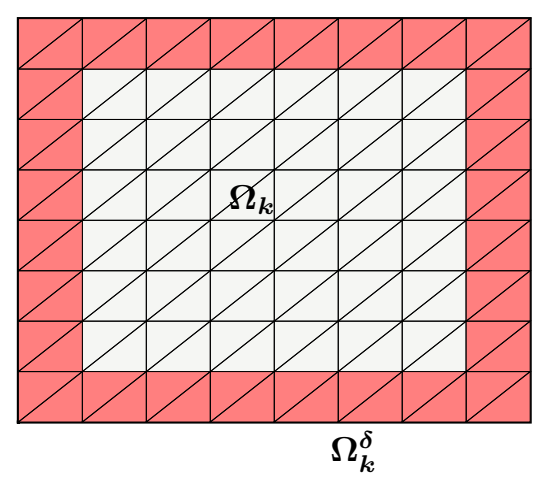

FIG. 2.1. $\Omega_{k}^{\delta}$ is the layer corresponding to the subdomain $\Omega_{k}$ and consisting of elements (triangles) of $T_{h}\left(\Omega_{k}\right)$ touching the subdomain boundary $\partial \Omega_{k}$.

Each subdomain inherits its triangulation $T_{h}\left(\Omega_{k}\right)=\left\{\tau \in T_{h}: \tau \subset \Omega_{k}\right\}$ from $T_{h}(\Omega)$. Consequently, we define the local finite element space $V^{h}\left(\Omega_{k}\right)$ as the space of functions of $V^{h}$ restricted to $\bar{\Omega}_{k}$ and

$$
V_{0}^{h}\left(\Omega_{k}\right)=V^{h}\left(\Omega_{k}\right) \cap H_{0}^{1}\left(\Omega_{k}\right) .
$$

Let $\Omega_{k}^{\delta} \subset \Omega_{k}, k=1, \ldots, N$, be the open discrete layers, where each $\Omega_{k}^{\delta}$ is defined as the interior of the sum of all closed triangles $\tau \in T_{h}\left(\Omega_{k}\right)$ such that $\partial \tau \cap \partial \Omega_{k} \neq \emptyset$; cf. Figure 2.1. We introduce the local maxima and minima of the coefficients over a subdomain and its layer as

$$
\begin{aligned}
\underline{\alpha}_{k} & :=\min _{x \in \Omega_{k}} \alpha, & \bar{\alpha}_{k} & :=\max _{x \in \Omega_{k}} \alpha, \\
\underline{\alpha}_{k, \delta} & :=\min _{x \in \Omega_{k}^{\delta}} \alpha, & \bar{\alpha}_{k, \delta} & :=\max _{x \in \Omega_{k}^{\delta}} \alpha .
\end{aligned}
$$

Let also $\Omega_{h}, \partial \Omega_{h}, \Omega_{k, h}, \partial \Omega_{k, h}$, and $\Gamma_{h}$ denote the sets of nodal points which are on the respective sets $\Omega, \partial \Omega, \Omega_{k}, \partial \Omega_{k}$, and $\Gamma$.

3. Additive Schwarz method. The method (cf. [28]) is constructed using the abstract scheme of the additive Schwarz method (ASM); cf., e.g., [34, 37]. Accordingly, for each local subproblem, the subspace corresponding to the subdomain $\Omega_{k}, V_{k} \subset V^{h}$, is defined as the space of functions of $V_{0}^{h}\left(\Omega_{k}\right)$ extended by zero to the rest of $\Omega$, i.e.,

$$
V_{k}=\left\{u \in V^{h}: u(x)=0 \quad x \notin \Omega_{k}\right\}, \quad k=1, \ldots, N .
$$

For the coarse problem we propose two different coarse spaces for the Schwarz method, $V_{0}^{\text {TYPE }} \subset V^{h}$ where TYPE is either II or I defined below in Section 4; cf. (4.4). The corresponding 
projections $P_{k}: V^{h} \rightarrow V_{k}$ are defined as

$$
a\left(P_{k} u, v\right)=a(u, v), \quad v \in V_{k}, \quad k=1, \ldots, N,
$$

and $P_{0}^{\mathrm{TYPE}}: V^{h} \rightarrow V_{0}^{\mathrm{TYPE}}$ as

$$
a\left(P_{0}^{\mathrm{TYPE}} u, v\right)=a(u, v), \quad v \in V_{0}^{\mathrm{TYPE}}, \quad \text { TYPE } \in\{\mathrm{II}, \mathrm{I}\} .
$$

Now following the Schwarz scheme we write the additive Schwarz operator $P^{\text {TYPE }}: V^{h} \rightarrow V^{h}$ as

$$
P^{\mathrm{TYPE}} u=P_{0}^{\mathrm{TYPE}} u+\sum_{k=1}^{N} P_{k} u, \quad \text { TYPE } \in\{\text { II, I }\} .
$$

We may replace the original problem (2.1) with the following problem; cf., e.g., [34, 37]:

$$
P^{\mathrm{TYPE}} u_{h}^{*}=g^{\mathrm{TYPE}}, \quad \text { TYPE } \in\{\mathrm{II}, \mathrm{I}\},
$$

where $g^{\mathrm{TYPE}}=g_{0}^{\mathrm{TYPE}}+\sum_{k} g_{k}$ with $g_{0}^{\mathrm{TYPE}}=P_{0}^{\mathrm{TYPE}} u_{h}^{*}$ and $g_{k}=P_{k} u_{h}^{*}$. The function on the right-hand side of (3.2) can be computed without knowing $u_{h}^{*}$; cf. [34, 37]. The condition number bounds for $P^{\text {TYPE }}$ are given in Theorem 5.1 in Section 5.

4. Coarse spaces and interpolation operators. We start by introducing two coarse spaces (cf. [28]) for the additive average Schwarz method; they correspond to TYPE = I and TYPE = II. Basically, both have the same classical additive average Schwarz coarse space which is defined as the range of the average interpolation operator $I_{0}: V^{h} \rightarrow V^{h}$ (cf. [3, 12, 26]),

$$
I_{0} u(x)= \begin{cases}u(x) & x \in \Gamma_{h}, \\ \bar{u}_{k} & x \in \Omega_{k, h}, \quad k=1, \ldots, N,\end{cases}
$$

where $\bar{u}_{k}=\frac{1}{n_{k}} \sum_{x \in \partial \Omega_{k, h}} u(x)$ with $n_{k}$ being the number of nodal points in $\partial \Omega_{k, h}$, in other words, the discrete average of $u$ over the boundary of the subdomain. This space is then enriched with functions that are adaptively selected eigenfunctions of a specially constructed generalized eigenvalue problem (cf. (4.2)) defined locally in each subdomain and extended by zero to the rest of the domain. This local generalized eigenvalue problem is of either $\mathrm{TYPE}=\mathrm{I}$ or TYPE $=\mathrm{II}$ differing in the bilinear form $b_{k}^{\mathrm{TYPE}}(\cdot, \cdot)$ used in (4.2). The resulting coarse space in either case is then the classical additive average Schwarz coarse space $I_{0} V^{h}$ enriched with the corresponding functions; cf. (4.2)-(4.4) below.

Here is how the generalized eigenvalue problem is defined locally in each subdomain $\Omega_{k}$. Find all eigenpairs: $\left(\lambda_{j}^{k, \text { TYPE }}, \psi_{j}^{k, \text { TYPE }}\right) \in\left(\mathbb{R}, V_{0}^{h}\left(\Omega_{k}\right)\right)$ such that

$$
\begin{aligned}
a_{k}\left(\psi_{j}^{k, \text { TYPE }}, v\right) & =\lambda_{j}^{k, \text { TYPE }} b_{k}^{\mathrm{TYPE}}\left(\psi_{j}^{k, \text { TYPE }}, v\right), \quad v \in V_{0}^{h}\left(\Omega_{k}\right), \\
b_{k}^{\mathrm{TYPE}}\left(\psi_{j}^{k, \text { TYPE }}, \psi_{j}^{k, \text { TYPE }}\right) & =1,
\end{aligned}
$$

where $a_{k}(\cdot, \cdot)$ and $b_{k}^{\mathrm{TPPE}}(\cdot, \cdot)$ for TYPE $\in\{\mathrm{I}, \mathrm{II}\}$ are symmetric bilinear forms defined as

$$
a_{k}(u, v):=\int_{\Omega_{k}} \alpha \nabla u \nabla v d x
$$

and

$$
\begin{aligned}
& b_{k}^{\mathrm{I}}(u, v):=\int_{\Omega_{k}} \underline{\alpha}_{k} \nabla u \nabla v d x, \\
& b_{k}^{\mathrm{II}}(u, v):=\int_{\Omega_{k}^{\delta}} \underline{\alpha}_{k, \delta} \nabla u \nabla v d x+\int_{\Omega_{k} \backslash \Omega_{k}^{\delta}} \alpha \nabla u \nabla v d x .
\end{aligned}
$$


Note that if two eigenvalues are different, then their respective eigenspaces and eigenfunctions are both $a_{k}(\cdot, \cdot)$ - and $b_{k}^{\mathrm{TYPE}}(\cdot, \cdot)$-orthogonal to each other. In case of an eigenvalue of multiplicity larger than one, we consider all its eigenfunctions as one. We also order the eigenvalues in decreasing order as $\lambda_{1}^{k, \text { TYPE }} \geq \lambda_{2}^{k, \text { TYPE }} \geq \ldots, \lambda_{N_{k}}^{k, \text { TYPE }}>0$, where $N_{k}$ is the dimension of $V_{0}^{h}\left(\Omega_{k}\right)$.

REMARK 4.1. We see that $1 \leq \lambda_{j}^{k, \mathrm{I}} \leq \frac{\bar{\alpha}_{k}}{\underline{\alpha}_{k}}$ and $1 \leq \lambda_{j}^{k, \mathrm{II}} \leq \frac{\bar{\alpha}_{k, \delta}}{\underline{\alpha}_{k, \delta}}$. Thus, when $\alpha$ is constant in $\Omega_{k}$, all eigenvalues of both eigenvalue problems are equal to one, and when $\alpha$ is constant in the layer $\Omega_{k}^{\delta}$, all eigenvalues $\lambda_{j}^{k, \text { II }}$ are equal to one.

For further use, we extend $\psi_{j}^{k, \text { TYPE }}$ by zero to the rest of the domain $\Omega$ denoting the extended function by the same symbol. Now let

$$
W_{k}^{\mathrm{TYPE}}:=\operatorname{Span}\left(\psi_{j}^{k, \mathrm{TYPE}}\right)_{j=1}^{M_{k}^{\mathrm{TYPE}}}, \quad \text { TYPE } \in\{\mathrm{II}, \mathrm{I}\},
$$

where $0 \leq M_{k}^{\text {TYP }}<N_{k}$ is a number either preset by the user or chosen adaptively (it is the number of eigenvalues whose magnitudes are smaller than or equal to a given threshold). We assume that if an eigenvalue which has been selected to be included has multiplicity larger than one, then all its eigenfunctions will be included in the $W_{k}^{\text {TYPE }}$. Consequently, $\lambda_{M_{k}^{\mathrm{TYE}}}^{\mathrm{TYPE}}+1<\lambda_{M_{k}^{\mathrm{TYPE}}}^{\mathrm{TYPE}}$. Thus $M_{k}^{\mathrm{TYPE}}=0$ means enrichment is not required in the subdomain $\Omega_{k}$. Our coarse spaces are then defined as

$$
V_{0}^{\mathrm{TYPE}}=I_{0} V^{h}+\sum_{k=1}^{N} W_{k}^{\mathrm{TYPE}}, \quad \text { TYPE } \in\{\mathrm{II}, \mathrm{I}\} .
$$

The two operators that we need for the analysis are defined here. The first one being a $b_{k}^{\mathrm{TPE}}(\cdot, \cdot)$-orthogonal projection operator $\Pi_{k}^{\mathrm{TYPE}}: V_{0}^{h}\left(\Omega_{k}\right) \rightarrow V_{0}^{h}\left(\Omega_{k}\right)$ given by

$$
\Pi_{k}^{\mathrm{TYPE}} v=\sum_{j=1}^{M_{k}^{\mathrm{TYPE}}} b_{k}^{\mathrm{TYPE}}\left(v, \psi_{j}^{k, \mathrm{TYPE}}\right) \psi_{j}^{k, \mathrm{TYPE}}, \quad \text { TYPE } \in\{\mathrm{II}, \mathrm{I}\},
$$

where $\left(\psi_{j}^{k \text {,TYPE }}\right)_{j}$ form the $b_{k}^{\text {TYPE }}(\cdot, \cdot)$-orthonormal eigenbasis of $V_{0}^{h}\left(\Omega_{k}\right)$; cf. (4.2). The second operator is defined in the following paragraph.

We note that for any $u \in V^{h}$, the function $w=u-I_{0} u \in V^{h}$ equals zero on $\partial \Omega_{k}$ for each $k$, thus the projection $\Pi_{k}^{\mathrm{TYPE}}\left(u-I_{0} u\right)_{\mid \Omega_{k}}$, TYPE $\in\{$ II, I $\}$, is properly defined; cf. (4.5). It is then further extended by zero to the rest of the domain obtaining a function in $W_{k}^{\text {TYPE}}$; cf. (4.3). Further, the extended projection $\Pi_{k}^{\mathrm{TYPE}}\left(u-I_{0} u\right)_{\mid \Omega_{k}}$ will be denoted by the same symbol. Then, $I_{0}^{\text {TYPE }}: V^{h} \rightarrow V_{0}^{\text {TYPE }}$ is defined as follows:

$$
I_{0}^{\mathrm{TYPE}}=I_{0} u+\sum_{k=1}^{N} \Pi_{k}^{\mathrm{TYPE}}\left(u-I_{0} u\right), \quad \text { TYPE } \in\{\text { II, I }\} .
$$

5. Condition number bound. We present our main theoretical result here, cf. Theorem 5.1, which gives an upper bound for the condition number of the preconditioned system (3.2).

THEOREM 5.1. Let $P^{\text {TYPE }}$ be the additive Schwarz operator, where TYPE $\in\{I I, I\}$, as defined in (3.1). Then for all $u \in V^{h}$

$$
\left(\min _{k} \frac{1}{\lambda_{M_{k}^{T Y P E}+1}^{T Y P E}}\right) \frac{h}{H} a(u, u) \lesssim a\left(P^{T Y P E} u, u\right) \lesssim a(u, u), \quad \text { TYPE } \in\{I I, I\},
$$


where $H=\max _{k=1, \ldots, N} \operatorname{diam}\left(\Omega_{k}\right)$ and $\lambda_{M_{k}^{T Y P E}+1}^{\text {TYPE }}$ is the $M_{k}^{\text {TYPE }+1}$-st eigenvalue of (4.2) (cf. also (4.3)).

Proof. The proof follows from the theory of the abstract Schwarz framework which requires that three key assumptions are satisfied; see, e.g., [34, 37]. The first assumption is the existence of a stable splitting (cf., e.g., [37, Assumption 2.2]), which will be proved in Lemma 5.5 below. It is straightforward to verify the other two assumptions. The local stability assumption is satisfied with the stability constant being equal to one since only the exact bilinear form is used for the local bilinear forms. While the assumption on the Cauchy-Schwarz relationship between the local subspaces is satisfied with the spectral radius of the matrix of constants of the strengthened Cauchy-Schwarz inequalities being equal to one since the local subspaces are orthogonal to each other.

We prove the stability assumption needed in the proof of Theorem 5.1 in Lemma 5.5. In order for that, we require a few estimates which we state first.

A spectral estimate. A well-known spectral estimate which is used in our analysis is presented here. Let $V$ be a finite-dimensional space with a symmetric positive definite bilinear form $b(u, v)$ and a symmetric non-negative definite form $a(u, v)$. We say that $\lambda \in \mathbb{R}$ is an eigenvalue of the following eigenvalue problem if there exists a non-zero eigenfunction $\phi_{\lambda} \in V$ such that

$$
a\left(\phi_{\lambda}, v\right)=\lambda b\left(\phi_{\lambda}, v\right), \quad v \in V .
$$

Now let $V_{\lambda}$ be the eigenspace associated with the eigenvalue $\lambda$, and let $\Pi_{\mu}$, for $\mu>0$, be the $b(\cdot, \cdot)$-orthogonal projection onto the space

$$
W_{\mu}=\sum_{\lambda>\mu} V_{\lambda}
$$

where the sum is taken over all eigenvalues greater than $\mu$ (a threshold). Then, we have the following lemma.

LEMMA 5.2. For $u \in V$, it holds that

$$
\left\|u-\Pi_{\mu} u\right\|_{a}^{2} \leq \mu\|u\|_{b}^{2},
$$

where $\|u\|_{b}=b(u, u)$ and $\|u\|_{a}=a(u, u)$.

Proof. The proof is quite standard, yet, for the completeness we give it here. If $\lambda_{k}$ and $\lambda_{l}$ are two distinct eigenvalues, then $V_{\lambda_{k}}$ are $V_{\lambda_{l}}$ are orthogonal to each other with respect to both $a(\cdot, \cdot)$ and $b(\cdot, \cdot)$. Let $u \in V$, then since $V=\sum_{\lambda} V_{\lambda}$ and the eigenspaces are both $b(\cdot, \cdot)$ and $a(\cdot, \cdot)$-orthogonal, we can uniquely write $u=\sum_{\lambda} \psi_{\lambda}$ with $\psi_{\lambda} \in V_{\lambda}$. Now using (5.1), we have

$$
\|u\|_{b}^{2}=\sum_{\lambda}\left\|\psi_{\lambda}\right\|_{b}^{2} \quad\|u\|_{a}^{2}=\sum_{\lambda}\left\|\psi_{\lambda}\right\|_{a}^{2}=\sum_{\lambda} \lambda\left\|\psi_{\lambda}\right\|_{b}^{2}
$$

Using the above and noting that $u-\Pi_{\mu} u=u-\sum_{\lambda>\mu} \psi_{\lambda}=\sum_{\lambda \leq \mu} \psi_{\lambda}$, we immediately get

$$
\left\|u-\Pi_{\mu} u\right\|_{a}^{2}=\sum_{\lambda \leq \mu}\left\|\psi_{\lambda}\right\|_{a}^{2}=\sum_{\lambda \leq \mu} \lambda\left\|\psi_{\lambda}\right\|_{b}^{2} \leq \sum_{\lambda \leq \mu} \mu\left\|\psi_{\lambda}\right\|_{b}^{2} \leq \sum_{\lambda} \mu\left\|\psi_{\lambda}\right\|_{b}^{2}=\mu\|u\|_{b}^{2},
$$

which ends the proof.

Now, applying the above lemma to the operator $\Pi_{k}^{\text {TYPE }}$, for TYPE $\in\{$ II, I $\}$, and taking $\mu=\lambda_{M_{k}^{\mathrm{TYE}}}^{\mathrm{TYPE}}+1$, we get the following estimate for the operator:

$$
a_{k}\left(u-\Pi_{k}^{\mathrm{TYPE}} u, u-\Pi_{k}^{\mathrm{TYPE}} u\right) \leq \lambda_{M_{k}^{\mathrm{TYPE}}+1}^{\mathrm{TYPE}} b_{k}^{\mathrm{TYPE}}(u, u), \quad \text { TYPE } \in\{\mathrm{II}, \mathrm{I}\} .
$$


An estimate of the coarse interpolation operator. What we need first is an estimate of the average interpolation operator $I_{0}$ in the norms induced by the two local bilinear forms $b_{k}^{\text {TYPE }}(\cdot, \cdot)$ for TYPE $\in\{$ II, I $\}$.

LEMMA 5.3. Let $I_{0}$ be the average interpolation operator as defined in (4.1). Then for all $u \in V^{h}$

$$
b_{k}^{\text {TYPE }}\left(u-I_{0} u, u-I_{0} u\right) \lesssim \frac{H}{h} a_{k}(u, u), \quad \text { TYPE } \in\{I I, I\} .
$$

Proof. In case of TYPE=I, the proof follows from [3] (see also [26]). Namely, we get

$$
b_{k}^{\mathrm{I}}\left(u-I_{0} u, u-I_{0} u\right)=\underline{\alpha}_{k}\left\|\nabla\left(u-I_{0} u\right)\right\|_{L^{2}\left(\Omega_{k}\right)}^{2} \lesssim \underline{\alpha}_{k} \frac{H}{h}\|\nabla u\|_{L^{2}\left(\Omega_{k}\right)}^{2} \leq \frac{H}{h} a_{k}(u, u) .
$$

For the first inequality above we refer to [3] while the second inequality follows from the definition of $\underline{\alpha}_{k}$; cf. (2.2).

In the case of TYPE=II we have $\nabla\left(u-I_{0} u\right)=\nabla u$ on each fine triangle $\tau \in T_{h}\left(\Omega_{k}\right)$ that is not inside $\Omega_{k}^{\delta}$, since $I_{0} u$ is constant there; cf. (4.1). Hence,

$$
\begin{aligned}
b_{k}^{\mathrm{II}}\left(u-I_{0} u, u-I_{0} u\right) & =\left\|\alpha_{k}^{1 / 2} \nabla\left(u-I_{0} u\right)\right\|_{L^{2}\left(\Omega_{k} \backslash \Omega_{k}^{\delta}\right)}^{2}+\underline{\alpha}_{k, \delta}\left\|\nabla\left(u-I_{0} u\right)\right\|_{L^{2}\left(\Omega_{k}^{\delta}\right)}^{2} \\
& =\left\|\alpha_{k}^{1 / 2} \nabla u\right\|_{L^{2}\left(\Omega_{k} \backslash \Omega_{k}^{\delta}\right)}^{2}+\underline{\alpha}_{k, \delta}\left\|\nabla\left(u-I_{0} u\right)\right\|_{L^{2}\left(\Omega_{k}^{\delta}\right)}^{2} \\
& \leq\left\|\alpha_{k}^{1 / 2} \nabla u\right\|_{L^{2}\left(\Omega_{k}\right)}^{2}+\underline{\alpha}_{k, \delta}\left\|\nabla\left(u-I_{0} u\right)\right\|_{L^{2}\left(\Omega_{k}^{\delta}\right)}^{2} \\
& =a_{k}(u, u)+\underline{\alpha}_{k, \delta}\left\|\nabla\left(u-I_{0} u\right)\right\|_{L^{2}\left(\Omega_{k}^{\delta}\right)}^{2} .
\end{aligned}
$$

To estimate the second term above, we utilize a triangle inequality and (2.2), giving

$$
\begin{aligned}
\underline{\alpha}_{k, \delta}\left\|\nabla\left(u-I_{0} u\right)\right\|_{L^{2}\left(\Omega_{k}^{\delta}\right)}^{2} & \lesssim \underline{\alpha}_{k, \delta}\|\nabla u\|_{L^{2}\left(\Omega_{k}^{\delta}\right)}^{2}+\underline{\alpha}_{k, \delta}\left\|\nabla I_{0} u\right\|_{L^{2}\left(\Omega_{k}^{\delta}\right)}^{2} \\
& \leq a_{k}(u, u)+\underline{\alpha}_{k, \delta}\left\|\nabla I_{0} u\right\|_{L^{2}\left(\Omega_{k}^{\delta}\right)}^{2} .
\end{aligned}
$$

Again, to estimate the second term in (5.3), we proceed analogously to the lines of the proof of Lemma 4.4 in [26], however, for the sake of completeness we provide a short proof here.

Note that for a triangle $\tau \in T_{h}$ we have the following equivalence,

$$
|\nabla u|_{L^{2}(\tau)}^{2} \lesssim \sum_{i, j \in\{1,2,3\}}\left|u\left(x_{i}\right)-u\left(x_{j}\right)\right|^{2} \lesssim|\nabla u|_{L^{2}(\tau)}^{2},
$$

where the sum is taken over all pairs of vertices of $\tau$. Using this and the discrete equivalence of the $L^{2}$-norm over a $1 \mathrm{D}$ element, we get

$$
\begin{aligned}
\left\|\nabla\left(I_{0} u\right)\right\|_{L^{2}\left(\Omega_{k}^{\delta}\right)}^{2} & =\sum_{\tau \subset \Omega_{k}^{\delta}}\left\|\nabla I_{0} u\right\|_{L^{2}(\tau)}^{2} \lesssim \sum_{x \in \partial \Omega_{k, h}}\left(u(x)-\bar{u}_{k}\right)^{2} \\
& \lesssim h^{-1}\left\|u-\bar{u}_{k}\right\|_{L^{2}\left(\partial \Omega_{k}\right)}^{2} .
\end{aligned}
$$

By a trace theorem, the Poincaré inequality, and a scaling argument, we get

$$
h^{-1}\left\|u-\bar{u}_{k}\right\|_{L^{2}\left(\partial \Omega_{k}\right)}^{2} \lesssim \frac{H}{h}\|\nabla u\|_{L^{2}\left(\Omega_{k}\right)}^{2} .
$$

Now using the last two estimates and (2.2), the second term on the right-hand side of (5.3) can be bounded as

$$
\underline{\alpha}_{k, \delta}\left\|\nabla\left(I_{0} u\right)\right\|_{L^{2}\left(\Omega_{k}^{\delta}\right)}^{2} \lesssim \underline{\alpha}_{k, \delta} \frac{H}{h}\|\nabla u\|_{L^{2}\left(\Omega_{k}\right)}^{2} \leq \frac{H}{h} a_{k}(u, u),
$$

which ends the proof. 
The following lemma gives a stability estimate for the coarse operator $I_{0}^{\text {TYPE }}$ (cf. (4.6)), where TYPE $\in\{\mathrm{I}, \mathrm{II}\}$.

LEMMA 5.4. Let $I_{0}^{\text {TYP }}$ be a coarse operator defined in (4.6). Then for $u \in V^{h}$

$$
a\left(u-I_{0}^{T Y P E} u, u-I_{0}^{T Y P E} u\right) \lesssim \max _{k} \lambda_{M_{k}^{T Y Y P E}+1}^{T Y E} \frac{H}{h} a(u, u), \quad T Y P E \in\{I I, I\},
$$

for any $u \in V^{h}$. Here $\lambda_{M_{k}^{T Y P E}+1}^{T Y P}$ is as in Theorem 5.1.

Proof. Define $w=u-I_{0} u$. Clearly, $w$ is equal to zero on the interface $\Gamma$. Note that

$$
u-I_{0}^{\mathrm{TYPE}} u=\sum_{k}\left(I-\Pi_{k}^{\mathrm{TYPE}}\right) w
$$

which is also equal to zero on the interface $\Gamma$. Then

$$
a\left(u-I_{0}^{\mathrm{TYPE}} u, u-I_{0}^{\mathrm{TPPE}} u\right)=\sum_{k} a_{k}\left(\left(I-\Pi_{k}^{\mathrm{TPPE}}\right) w,\left(I-\Pi_{k}^{\mathrm{TYPE}}\right) w\right) .
$$

Using (5.2) and Lemma 5.3, we get

$$
a_{k}\left(\left(I-\Pi_{k}^{\mathrm{TYPE}}\right) w,\left(I-\Pi_{k}^{\mathrm{TYPE}}\right) w\right) \lesssim \lambda_{M_{k}^{\mathrm{TYPE}}+1}^{\mathrm{TY}} b_{k}^{\mathrm{TPE}}(w, w) \lesssim \lambda_{M_{k}^{\mathrm{TYPE}}+1}^{\mathrm{TPE}} \frac{H}{h} a_{k}(u, u) .
$$

Summing over all subdomains ends the proof.

We are now ready to give a proof of the stability assumption of the splitting needed for the proof in Theorem 5.1.

LEMMA 5.5 (Stable Splitting). For $u \in V^{h}$, let $u_{0}=I_{0}^{\text {TYPE }} u \in V_{0}^{\text {TYPE }}$, for TYPE $\in\{I I, I\}$, and let $u_{k} \in V_{k}$, for $k=1, \ldots, N$, equal to $\left(u-u_{0}\right)_{\mid \Omega_{k}}$ extended by zero to the rest of the domain. Then $u=u_{0}+\sum_{k=1}^{N} u_{i}$, and

$$
a\left(u_{0}, u_{0}\right)+\sum_{k=1}^{N} a\left(u_{k}, u_{k}\right) \lesssim \max _{k} \lambda_{M_{k}^{T Y P E}}^{\text {TYPE }}+\frac{H}{h} a(u, u), \quad \text { TYPE } \in\{I I, I\} .
$$

Proof. It is not difficult to see that the splitting $u=u_{0}+\sum_{k=1}^{N} u_{i}$ is valid following the definition of $u_{k}$. We prove the stability of this splitting as follows. Using a triangle inequality we get

$$
a\left(u_{0}, u_{0}\right) \lesssim a(u, u)+a\left(u-I_{0}^{\mathrm{TYPE}} u, u-I_{0}^{\mathrm{TYPE}} u\right)
$$

Noting that

$$
a\left(u_{k}, u_{k}\right)=a_{k}\left(u_{k}, u_{k}\right)=a_{k}\left(u-I_{0}^{\mathrm{TYPE}} u, u-I_{0}^{\mathrm{TYPE}} u\right), \quad k=1, \ldots, N,
$$

we have

$$
\sum_{k=1}^{N} a\left(u_{k}, u_{k}\right)=\sum_{k=1}^{N} a_{k}\left(u-I_{0}^{\mathrm{TYPE}} u, u-I_{0}^{\mathrm{TYPE}} u\right)=a\left(u-I_{0}^{\mathrm{TYPE}} u, u-I_{0}^{\mathrm{TYPE}} u\right) .
$$

Now, combining (5.4) and (5.5) and then applying Lemma 5.4, we obtain the proof. 


\section{ETNA}

Kent State University and

Johann Radon Institute (RICAM)

6. Numerical experiments. We present some simple numerical experiments to validate our theory. The problem is considered on a unit square with homogeneous boundary conditions and the right-hand side function $f(x)=2 \pi^{2} \sin (\pi x) \sin (\pi y)$. We chose the following distribution of the coefficient $\alpha$ for the experiment: it consists of a background, channels crossing inside subdomains and stretching out of subdomains (continuous across) or stopping at subdomain boundaries (discontinuous across), and inclusions along subdomain boundaries (placed at the corners); see Figure 6.1 for an illustration with $3 \times 3$ subdomains. $\alpha$ is equal to $\alpha_{b}$ in the background, $\alpha_{c}$ in the crossing channels, and $\alpha_{i}$ in the inclusions placed at the corners. Experiments have been performed using the proposed methods as preconditioners with a conjugate gradients iteration and stopping the iterations when the residual norm in each case is reduced by the factor 5e-6. The multiplicative version (cf. [32]) of the preconditioner has also been used, the results of which are reported for comparison. As expected, cf., e.g., [32], the multiplicative version converges twice as fast as the additive version in terms of the number of iterations, its condition number being one-fourth of that of the additive version. For an efficient implementation of the multiplicative algorithm, we refer to the same paper.
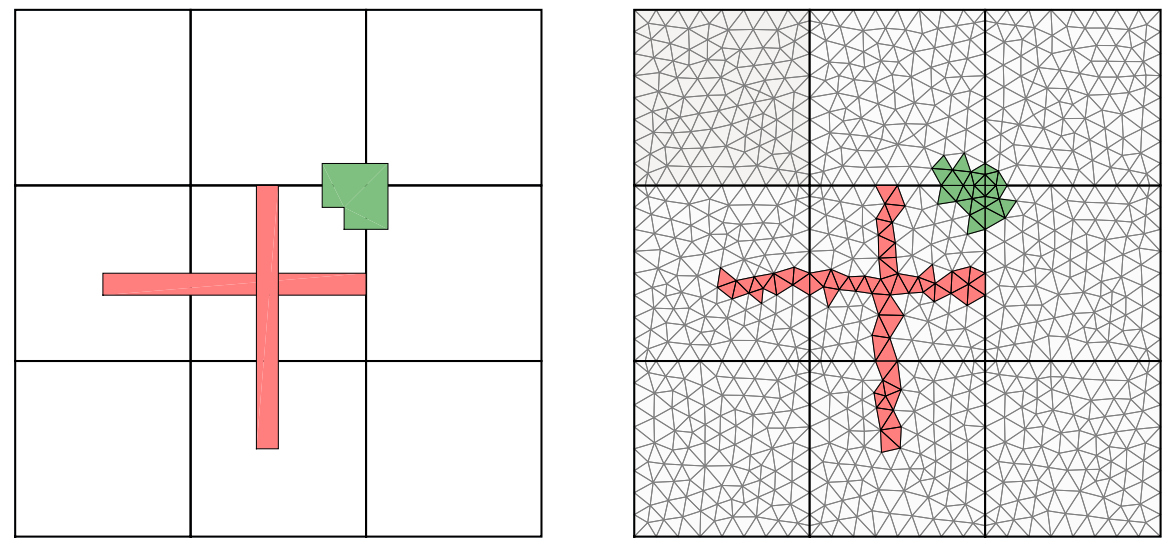

FIG. 6.1. On the left, a domain comprising of $3 \times 3$ subdomains with one inclusion (shaded green) placed at a subdomain corner and a pair of channels (shaded red) crossing each other inside a subdomain, and they are both either continuous or discontinuous across the subdomain's boundary. On the right, a finite element triangulation of the domain showing the corresponding channels and the inclusion in the triangulation. $\alpha$ is equal to $\alpha_{b}$ in the background, $\alpha_{c}$ in the crossing channels, and $\alpha_{i}$ in the inclusions.

In our first experiment, we study the convergence behavior of the proposed method as we vary the mesh size parameters $H$ and $h$ and the jump in the coefficient $\alpha$, while for the enrichment, we include only those eigenfunctions whose eigenvalues are greater than a given threshold (adaptive enrichment). We refer to Table 6.1, where the number of iterations required to converge and a condition number estimate (in parentheses) for each experiment are given for the TYPE $=$ II algorithm.

The results of TYPE $=$ I algorithm have been very similar to those of TYPE $=$ II, however, there has been a significant difference in the number of eigenfunctions included between the two, which we will discuss later; cf. Figure 6.2. The threshold for the eigenvalues has been set to 100 . 


\section{ETNA}

Kent State University and

Johann Radon Institute (RICAM)

TABLE 6.1

The number of iterations and a condition number estimate (in parentheses) for varying $H$ and $h$. The left block of results correspond to the additive version $(A D D)$ while the right block corresponds to the multiplicative version (MLT) of the average Schwarz method. The first three rows correspond to the coefficient distribution $\alpha_{b}=1$, $\alpha_{c}=1 \mathrm{e} 2$, and $\alpha_{i}=1 \mathrm{e} 4$, and the last three rows correspond to the coefficient distribution $\alpha_{b}=1, \alpha_{c}=1 \mathrm{e} 4$, and $\alpha_{i}=1 \mathrm{e} 6$.

\begin{tabular}{c|ccc|ccc}
\multicolumn{1}{c|}{} & \multicolumn{3}{c|}{ Additive (ADD) } & \multicolumn{3}{c}{ Multiplicative (MLT) } \\
\hline $1 / 18$ & $1 / 3$ & $1 / 6$ & $1 / 9$ & $1 / 3$ & $1 / 6$ & $1 / 9$ \\
$1 / 36$ & $54(5.84 \mathrm{e} 1)$ & & & $17(1.49 \mathrm{e} 1)$ & & \\
$1 / 54$ & $70(1.35 \mathrm{e} 2)$ & $52(5.71 \mathrm{e} 1)$ & & $28(3.40 \mathrm{e} 1)$ & $26(1.45 \mathrm{e} 1)$ & \\
\hline $1 / 18$ & $37(5.80 \mathrm{e} 1)$ & $67(9.32 \mathrm{e} 1)$ & $58(6.03 \mathrm{e} 1)$ & $35(5.34 \mathrm{e} 1)$ & $34(2.36 \mathrm{e} 1)$ & $29(1.53 \mathrm{e} 1)$ \\
$1 / 36$ & $53(1.34 \mathrm{e} 2)$ & $53(5.60 \mathrm{e} 1)$ & & $19(1.48 \mathrm{e} 1)$ & & \\
$1 / 54$ & $67(2.12 \mathrm{e} 2)$ & $68(9.19 \mathrm{e} 1)$ & $59(5.94 \mathrm{e} 1)$ & $33(5.33 \mathrm{e} 1)$ & $34(2.32 \mathrm{e} 1)$ & $29(1.51 \mathrm{e} 1)$
\end{tabular}

TABLE 6.2

The number of iterations and a condition number estimate (in parentheses) for a fixed number of eigenfunctions for the enrichment (respectively, 0, 2, 4, 5, 6, 7 of eigenfunctions in each subdomain). The first line (ADD) of results correspond to the additive version while the second line $(M L T)$ corresponds to the multiplicative version of the method. Here $H=1 / 6, h=1 / 36, \alpha_{b}=1, \alpha_{c}=1 \mathrm{e} 4$, and $\alpha_{i}=1 \mathrm{e} 6$.

\begin{tabular}{c|cccccc} 
& 0 & 2 & 4 & 5 & 6 & 7 \\
\hline ADD & $508(3.09 \mathrm{e} 6)$ & $431(1.08 \mathrm{e} 6)$ & $186(1.98 \mathrm{e} 4)$ & $61(4.62 \mathrm{e} 2)$ & $49(4.78 \mathrm{e} 1)$ & $48(4.71 \mathrm{e} 1)$ \\
MLT & $259(7.73 \mathrm{e} 5)$ & $218(2.71 \mathrm{e} 5)$ & $94(4.96 \mathrm{e} 3)$ & $30(1.15 \mathrm{e} 3)$ & $24(1.22 \mathrm{e} 1)$ & $24(1.20 \mathrm{e} 1)$
\end{tabular}

The columns of Table 6.1 correspond to the subdomain size $H$ and the rows correspond to the mesh size $h$. In order to have the same pattern in the distribution of $\alpha$ even when we vary the size of the subdomains, that is to have crossing channels inside subdomains and inclusions at subdomain corners as illustrated in Figure 6.1, we let the size of the channels and the inclusions vary proportionally with $H$, which is somewhat artificial but inevitable for the purpose of this experiment. We should, however, mention that they do not vary with the mesh size $h$ so that each column of the table corresponds to the same set of channels and inclusions. We note that the diagonal entries of the table correspond to the same mesh size ratio $\frac{H}{h}$. The corresponding condition number estimates as seen from the table are very close to each other suggesting that the proposed preconditioners are scalable in the sense that the condition number varies proportionately with the size of the subproblem, i.e., the ratio $\frac{H}{h}$. The fact that the condition number is independent of the jumps is also evident from the table.

Following our analysis, there is a minimum number of eigenfunctions (corresponding to the bad eigenvalues) which should be added in the enrichment for the method to be robust with respect to the contrast. In order to see that, in our next experiment, we choose one particular discretization $(H, h)$ and distribution of $\alpha$ and run our algorithm each time with a fixed number of eigenfunctions for all subdomains. For the experiment we have chosen $H=1 / 6$ (i.e., $6 \times 6$ subdomains) and $h=1 / 36$, and the results are presented in Table 6.2. As we can see from the table, the condition number improves as more and more eigenfunctions are included in the enrichment but stops (or improves very slowly) once the sixth eigenfunction has been included. So the minimum number of eigenfunctions, in this case, is six. This also agrees with the adaptive version, cf. Table 6.1, where the same test case needed six eigenfunctions.

In our final experiment, we compare the two algorithms, corresponding to TYPE $=$ II and TYPE $=\mathrm{I}$. Although they are very similar in their convergence behavior (their condition number estimates are very close to each other), the former requires far less eigenvalues in order to achieve the same level of convergence whenever there are inclusions or channels inside a 


\section{ETNA}

Kent State University and

Johann Radon Institute (RICAM)

subdomain (cf. Figure 6.2) suggesting that the TYPE = II algorithm has a clear advantage over the other in such cases.

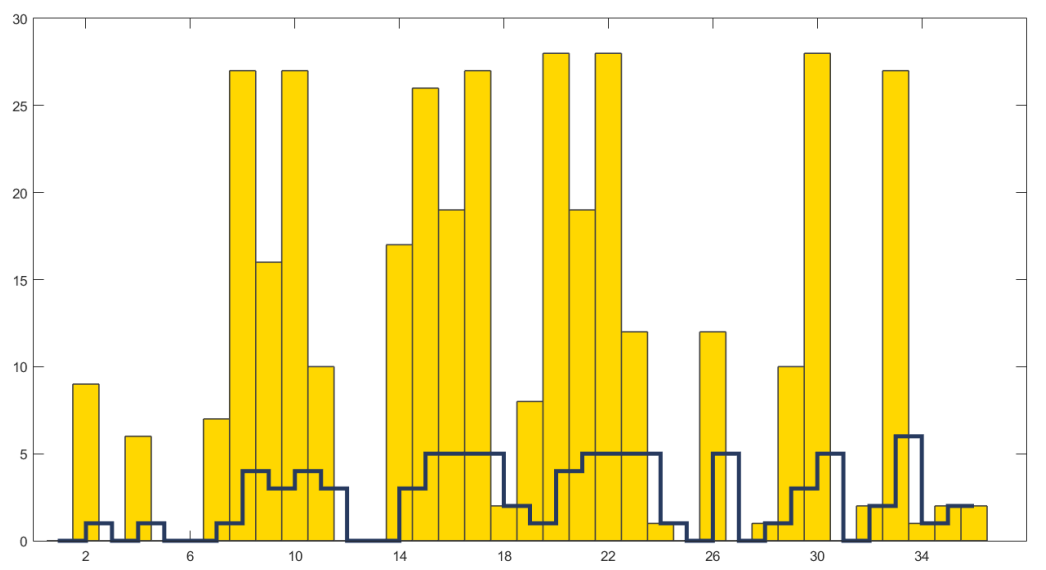

FIG. 6.2. The number of eigenvalues in each subdomain (36 subdomains) with values larger than 100 that have been selected for the enrichment. Here $H=1 / 6$ (i.e., $6 \times 6$ subdomains) and $h=1 / 36, \alpha_{b}=1, \alpha_{c}=1 \mathrm{e} 4$, and $\alpha_{i}=1 \mathrm{e} 6$. The bar graph corresponds to $\mathrm{TYPE}=\mathrm{I}$ algorithm, and the stair graph corresponds to $\mathrm{TYPE}=\mathrm{II}$ algorithm.

Acknowledgments. The first author was partially supported by Polish Scientific Grant 2016/21/B/ST1/00350.

\section{REFERENCES}

[1] J. R. AARnES AND T. Y. HoU, Multiscale domain decomposition methods for elliptic problems with high aspect ratios, Acta Math. Appl. Sin. Engl. Ser., 18 (2002), pp. 63-76.

[2] P. E. BJøRSTAD, M. DRYja, AND T. RAhman, Additive Schwarz methods for elliptic mortar finite element problems, Numer. Math., 95 (2003), pp. 427-457.

[3] P. E. BJøRSTAD, M. DRYJA, AND E. VAINIK Ko, Additive Schwarz methods without subdomain overlap and with new coarse spaces, in Domain Decomposition Methods in Sciences and Engineering (Beijing, 1995), R. Glowinski, J. Périaux, Z.-C. Shi, and O. Widlund, eds., Wiley, Chichester, 1997, pp. 141-157.

[4] P. BJøRSTAD, J. Koster, AND P. KRZYŻANOWSKI, Domain decomposition solvers for large scale industrial finite element problems, in Applied Parallel Computing. New Paradigms for HPC in Industry and Academia, T. Sørevik, F. Manne, A. H. Gebremedhin, and R. Moe, eds., vol. 1947 of Lecture Notes in Comput. Sci., Springer, Berlin, 2001, pp. 373-383.

[5] P. BJøRSTAD AND P. KRZYŻANOWSKI, A flexible 2-level Neumann-Neumann method for structural analysis problems, in Parallel Processing and Applied Mathematics, R. Wyrzykowski, J. Dongarra, M. Paprzycki, and J. Waśniewski, eds., vol. 2328 of Lecture Notes in Comput. Sci., Springer, Berlin, 2002, pp. 387-394.

[6] D. Braess, Finite Elements, 3rd. ed., Cambridge University Press, Cambridge, 2007.

[7] S. C. BREnNER And L. R. ScotT, The Mathematical Theory of Finite Element Methods, 3rd. ed., Springer, New York, 2008.

[8] J. G. CALVO AND O. B. WIDLUND, An adaptive choice of primal constraints for BDDC domain decomposition algorithms, Electron. Trans. Numer. Anal., 45 (2016), pp. 524-544. http://etna.ricam.oeaw.ac.at/vol.45.2016/pp524-544.dir/pp524-544.pdf

[9] T. Chartier, R. D. Falgout, V. E. Henson, J. Jones, T. Manteuffel, S. McCormick, J. Ruge, AND P. S. VASSILEVSKI, Spectral AMGe ( $\rho A M G e$ ), SIAM J. Sci. Comput., 25 (2003), pp. 1-26.

[10] E. Chung, Y. EFENDIEv, AND T. Y. Hou, Adaptive multiscale model reduction with generalized multiscale finite element methods, J. Comput. Phys., 320 (2016), pp. 69-95.

[11] V. Dolean, F. NATAF, R. SCHEICHL, AND N. SpIllane, Analysis of a two-level Schwarz method with 


\section{ETNA}

Kent State University and Johann Radon Institute (RICAM)

coarse spaces based on local Dirichlet-to-Neumann maps, Comput. Methods Appl. Math., 12 (2012), pp. 391-414.

[12] M. DRYJA AND M. SARKIS, Additive average Schwarz methods for discretization of elliptic problems with highly discontinuous coefficients, Comput. Methods Appl. Math., 10 (2010), pp. 164-176.

[13] Y. EfendieV, J. Galvis, R. LaZArov, S. MARgenov, AND J. Ren, Robust two-level domain decomposition preconditioners for high-contrast anisotropic flows in multiscale media, Comput. Methods Appl. Math., 12 (2012), pp. 415-436.

[14] Y. Efendiev, J. Galvis, R. LaZARov, AND J. Willems, Robust domain decomposition preconditioners for abstract symmetric positive definite bilinear forms, ESAIM Math. Model. Numer. Anal., 46 (2012), pp. 1175-1199.

[15] X. FEng AND T. RAhman, An additive average Schwarz method for the plate bending problem, J. Numer. Math., 10 (2002), pp. 109-125.

[16] J. GAlvis AND Y. EFENDIEv, Domain decomposition preconditioners for multiscale flows in high-contrast media, Multiscale Model. Simul., 8 (2010), pp. 1461-1483.

[17] — Domain decomposition preconditioners for multiscale flows in high contrast media: reduced dimension coarse spaces, Multiscale Model. Simul., 8 (2010), pp. 1621-1644.

[18] M. J. Gander, A. Loneland, AND T. RAhman, Analysis of a new harmonically enriched multiscale coarse space for domain decompostion methods, Preprint on arXiv, 2015. Submitted. https://arxiv.org/abs/1512.05285

[19] I. G. Graham, P. O. Lechner, And R. Scheichl, Domain decomposition for multiscale PDEs, Numer. Math., 106 (2007), pp. 589-626.

[20] H. H. KIM AND E. CHUNG, A BDDC algorithm with enriched coarse spaces for two-dimensional elliptic problems with oscillatory and high contrast coefficients, Multiscale Model. Simul., 13 (2015), pp. 571593.

[21] H. H. KIM, E. CHUNG, AND J. WANG, BDDC and FETI-DP preconditioners with adaptive coarse spaces for three-dimensional elliptic problems with oscillatory and high contrast coefficients, J. Comput. Phys., 349 (2017), pp. 191-214.

[22] A. KLAWONn, M. KÜHn, AND O. RHEINBACH, Adaptive coarse spaces for FETI-DP in three dimensions, SIAM J. Sci. Comput., 38 (2016), pp. A2880-A2911.

[23] A. KLAwONn, P. RADTKE, AND O. RHEInBACH, FETI-DP methods with an adaptive coarse space, SIAM J. Numer. Anal., 53 (2015), pp. 297-320.

[24] A. Klawonn, P. RadtKe, And O. Rheinbach, A comparison of adaptive coarse spaces for iterative substructuring in two dimensions, Electron. Trans. Numer. Anal., 45 (2016), pp. 75-106. http://etna.ricam.oeaw.ac.at/vol.45.2016/pp75-106.dir/pp75-106.pdf

[25] A. LONELAND, L. MARCINKOWSKI, AND T. RAHMAN, Additive average Schwarz method for a CrouzeixRaviart finite volume element discretization of elliptic problems, in Domain Decomposition Methods in Science and Engineering XXII, T. Dickopf, M. J. Gander, L. Halpern, R. Krause, and L. F. Pavarino, eds., vol. 104 of Lect. Notes Comput. Sci. Eng., Springer, Cham, 2016, pp. 587-594.

[26] _ - Additive average Schwarz method for a Crouzeix-Raviart finite volume element discretization of elliptic problems with heterogeneous coefficients, Numer. Math., 134 (2016), pp. 91-118.

[27] J. MANDEL AND B. SousedíK, Adaptive selection of face coarse degrees of freedom in the BDDC and the FETI-DP iterative substructuring methods, Comput. Methods Appl. Mech. Engrg., 196 (2007), pp. 1389-1399.

[28] L. MARCINKOWSKI AND T. RAHMAN, Two new enriched multiscale coarse spaces for additive average Schwarz method, in Domain Decomposition Methods in Science and Engineering XXIII, C.-O. Lee, X.-C. Cai, D. Keyes, H. Kim, A. Klawonn, E.-J. Park, and O. Widlund, eds., vol. 116 of Lect. Notes Comput. Sci. Eng., Springer, Cham, 2017, pp. 389-396.

[29] F. Nataf, H. Xiang, V. Dolean, and N. Spillane, A coarse space construction based on local Dirichletto-Neumann maps, SIAM J. Sci. Comput., 33 (2011), pp. 1623-1642.

[30] D.-S. OH, O. B. WidLund, S. ZAMPINI, AND C. R. Dohrmann, BDDC Algorithms with deluxe scaling and adaptive selection of primal constraints for Raviart-Thomas vector fields, Math. Comp., 87 (2018), pp. 659-692.

[31] C. PeChSTEIN And C. R. Dohrmann, A unified framework for adaptive BDDC, Electron. Trans. Numer. Anal., 46 (2017), pp. 273-336.

http://etna.ricam.oeaw.ac.at/vol.46.2017/pp273-336.dir/pp273-336.pdf

[32] T. RAHMAN, Schwarz preconditioned CG algorithm for the mortar finite element, Numer. Algorithms, 58 (2011), pp. 235-260.

[33] T. RAhman, X. XU, AND R. Hoppe, Additive Schwarz methods for the Crouzeix-Raviart mortar finite element for elliptic problems with discontinuous coefficients, Numer. Math., 101 (2005), pp. 551-572.

[34] B. Smith, P. BJøRSTAD, AND W. Gropp, Domain Decomposition: Parallel Multilevel Methods for Elliptic Partial Differential Equations, Cambridge University Press, Cambridge, 1996. 
[35] B. Sousedík, J. ŠísteK, AND J. MANDEL, Adaptive-multilevel BDDC and its parallel implementation, Computing, 95 (2013), pp. 1087-1119.

[36] N. Spillane, V. Dolean, P. Hauret, F. Nataf, C. Pechstein, and R. Scheichl, abstract robust coarse spaces for systems of PDEs via generalized eigenproblems in the overlaps, Numer. Math., 126 (2014), pp. 741-770.

[37] A. Toselli AND O. WidLund, Domain Decomposition Methods-Algorithms and Theory, Springer, Berlin, 2005. 